\title{
Fatigue crack growth in natural rubber: the role of SIC investigated through post-mortem analysis of fatigue striations
}

\author{
B. Ruellan ${ }^{\mathrm{a}, \mathrm{b}, \mathrm{c}}$, J.-B. Le Cam ${ }^{\mathrm{a}, \mathrm{c}, 1}$, E. Robin ${ }^{\mathrm{a}, \mathrm{c}}$, I. Jeanneau ${ }^{\mathrm{b}, \mathrm{c}}$, \\ F. Canévet ${ }^{\mathrm{b}, \mathrm{c}}$, G. Mauvoisin ${ }^{\mathrm{d}}$, D. Loison ${ }^{\mathrm{a}}$ \\ ${ }^{a}$ Univ Rennes, CNRS, IPR (Institute of Physics Rennes) - UMR 6251, F-35000 \\ Rennes, France \\ ${ }^{\mathrm{b}}$ Cooper Standard, 194 route de Lorient, 35043 Rennes - France. \\ ${ }^{\mathrm{c}}$ LC-DRIME, Joint Research Laboratory, Cooper Standard - Institut de Physique \\ UMR 6251, Campus de Beaulieu, Bât. 10B, F-35042 Rennes Cedex, France. \\ ${ }^{\mathrm{d}}$ Laboratoire de Génie Civil et Génie Mécanique EA 3913, IUT-Université de \\ Rennes 1, France
}

\begin{abstract}
Fatigue striations are investigated in order to provide additional information on the role of SIC in the fatigue crack growth resistance in natural rubber (NR). Uniaxial fatigue tests were carried out with Diabolo samples under a wide range of loading ratios and loading levels. Fracture surfaces were analyzed by using SEM. Two striation regimes were identified: small striation patches with different orientations (Regime 1) and zones with large and well formed striations, where wrenchings are no longer observed (Regime 2). As fatigue striations were observed for all the loading ratios applied, they are therefore not the signature of SIC in the lifetime reinforcement. Nevertheless, increasing the minimum value of the strain amplified the striation phenomenon and the occurrence of Regime 2. Results obtained have been qualitatively put into perspective with crack propagation curves provided in [17]. The analysis carried out unifies the results obtained in the literature for relaxing and fully relaxing loadings $\left(\mathrm{R}_{\varepsilon} \leq 0\right)$ in the sense that increasing the loading, i.e. the tearing energy, leads to an increase in the crack growth rate and to a striation typology evolution, especially the striation size. For non-relaxing loadings, our results suggest that the striation typology may be governed by the loading ratio rather than the crack growth rate. Finally, as striations did not appear when the tests were performed at temperature superior or equal to $90^{\circ} \mathrm{C}$, the formation of striation requires therefore that the material is crystallizing.
\end{abstract}


Key words: NR, strain-induced crystallization, fatigue, striations, lifetime reinforcement. 


\section{Introduction}

Since the beginning of the $20^{\text {th }}$ century, rubber-like materials have been widely used in many applications for their damping properties and their resistance to fatigue. As soon as 1925, the main phenomena involved in the mechanical response of rubber were characterized; non-exhaustively the entropic response $[9,10]$, the mechanical response including stress softening [5], the ability of the natural rubber to crystallize under tension [11]. Fatigue resistance was investigated from the 1940's after the pioneering work by Cadwell et al.[6] and Fielding [7] and more than 20 years later by Beatty [3]. Fatigue crack growth resistance of natural rubber was finally investigated through crack growth rate curves at different loading ratios $^{2}$. Typically, it was shown that non-relaxing loadings decrease the fatigue crack growth rate and increase the tearing energy below which no crack growth occurs [17]. Also, Mars has shown that the fracture mechanical behaviors associated with crystallization can be transformed into the Haigh and Cadwell spaces [19]. However, fatigue damage and resistance mechanisms, especially in crystallizable rubbers, remained not well understood, which is a real obstacle to fatigue lifetime prediction and improvement of rubber part design. This explains the upsurge of experimental studies since the last two decades. They aim at investigating and establishing the damage mechanisms (see for instance [24] and [12]) and the factors that affect the fatigue life [20]. Among the different phenomena and factors involved, multiaxiality, strain-induced crystallization (SIC), environmental temperature, thermal and thermo-oxidative ageing appear as the major ones. A number of them remains misunderstood and further investigations are still required, especially on SIC. Indeed, in case of crystallizable natural rubber (NR), the material submitted to cyclic loadings exhibits a high duration life and a strong lifetime reinforcement for non relaxing loadings (typically for uni-axial tension-tension loadings, when the minimum force $F_{\min }$ is superior to zero). This was observed as soon as 1940 by Cadwell and co-workers in NR, but this was not observed in non-crystallizable rubbers. This is the reason why such extraordinary properties were assumed to be due to SIC. To go further on the mechanisms of fatigue resistance and lifetime reinforcement due to SIC, crack propagation was investigated by coupling fatigue tests and post-mortem analyses $[24,14,8]$ at the microscopic scale. The observation of the failure surfaces have shown two different morphological details in crystallizable NR: wrenchings and striations [16]. As they were not observed on the failure surface of non-crystalizable rubbers, both were assumed to be due to SIC. They should therefore be the signature of the resistance

$\overline{1}$ Corresponding author jean-benoit.lecam@univ-rennes1.fr Fax : (+33) 223236111

2 defined as the minimum loading over the maximum loading 
to crack growth due to SIC at the microscopic scale.

Concerning wrenchings, their formation mechanism has been established by Le Cam et al. [14] and the role of SIC in the crack growth resistance has been further understood: more crystallized ligaments form between elliptical zones where the crack propagates and thus delay the crack propagation. Concerning fatigue striations, a very few studies have been carried out $[13,8,22,16]$ and the role of SIC on their formation has not been investigated and understood for all the loading conditions, typically under non-relaxing tension-tension loadings for which the lifetime reinforcement is observed. Studies available in the literature are summarized in the first part of the paper, where the difficulties in comparing them and the numerous questions they arise are pointed out. To go further on the role of SIC in fatigue crack growth resistance in NR, a new study of fatigue striations has been carried out. It is presented in the second part of the paper. Concluding remarks close the paper.

\section{State of art on fatigue striations}

Fatigue striations were observed and described in four studies, published from 2010 to 2013 [13, 8, 22, 16]. Only one was fully dedicated to fatigue striations [13], in case of relaxing loading conditions. Note that this paper is issued from the $\mathrm{PhD}$ thesis by Le Cam [12]. The present state of the art aims at summarizing these studies with respect to the materials studied, the sample geometries, the loading conditions and the results obtained.

\subsection{Experiments carried out}

The experimental conditions reported in the different studies are summarized in Table 1 . In this table, the ratio $\mathrm{R}$ is defined as the ratio of the minimum to the maximum loading, in terms of force $\left(\mathrm{R}_{F}\right)$ or displacement $\left(\mathrm{R}_{U}\right)$.

The materials used by the authors were NRs mainly filled with carbon black from 30 to $45 \mathrm{phr}^{3}$. It should be noted that Muñoz-Mejia [22] also investigated a NR filled with silica (50 and $60 \mathrm{phr}$ ). Even though the NRs used were all cis-1,4 poly-isoprene rubbers, the other chemical components strongly differed in amount

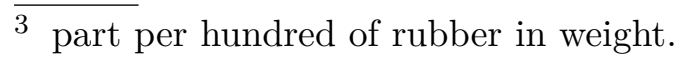




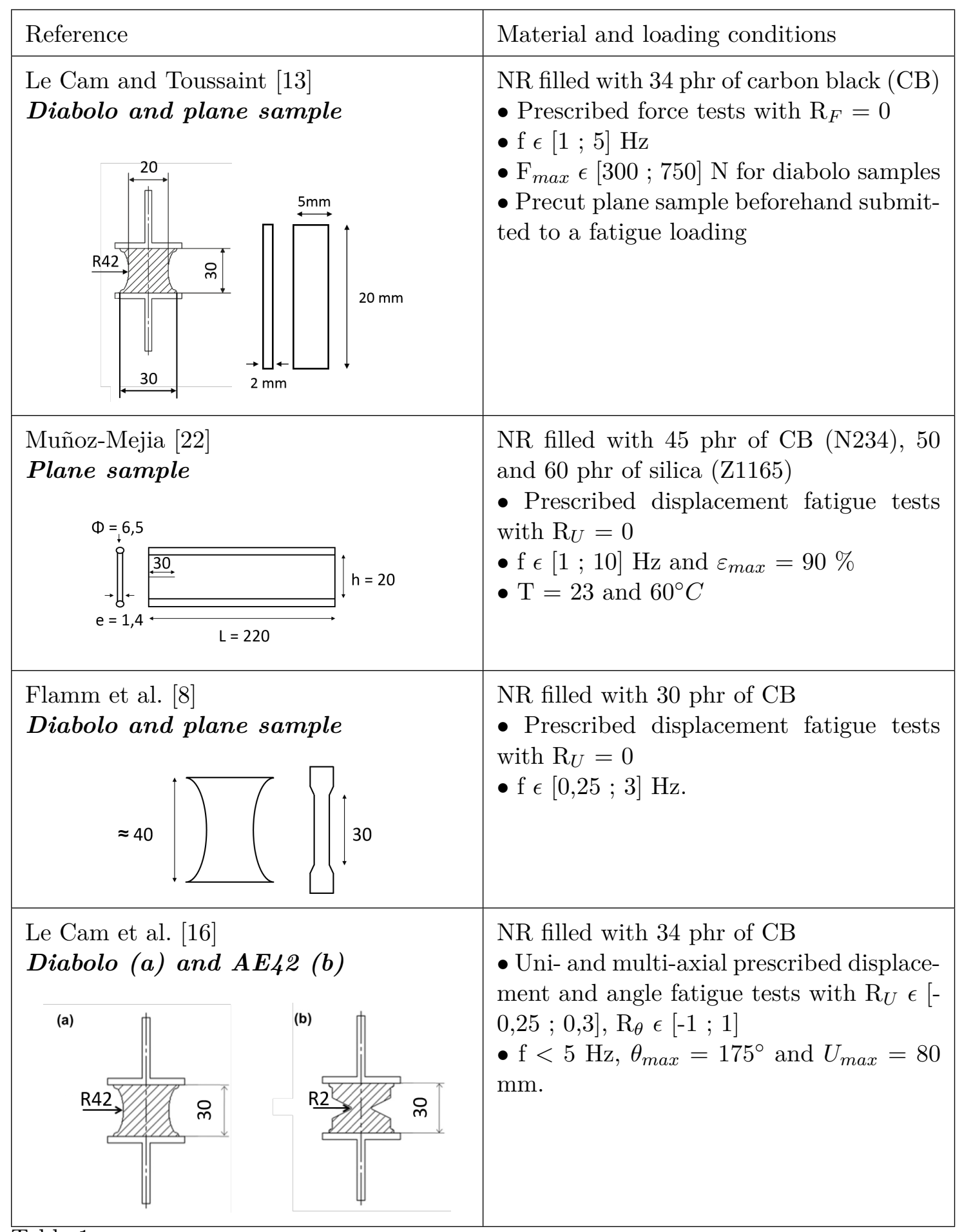

Table 1

Summary of experimental conditions. The dimensions are given in $\mathrm{mm}$. 
and nature from one formulation to another. Consequently, generalizing results to fatigue of filled NR is delicate.

\subsubsection{Sample geometries}

Sample geometries used were also different. Two types of sample geometry were found:

- Diabolo sample $[13,8,16]$ : this geometry was firstly introduced by Beatty [3]. Such geometry is well adapted to fatigue since no buckling is induced under compression and fatigue damage generally occurs at the surface of the sample, at half of its height, due to stress and strain concentrations. The volume of the matter tested is representative of industrial anti-vibratory parts,

- a plane sample used by Le Cam and Toussaint [13], Flamm et al. [8], Munoz et al. [21]. It enables the authors to follow and visualize the crack tip during propagation, which is not possible with the Diabolo sample. Nevertheless, the mechanical state at the crack tip of plane samples is very different from that in rubber parts, which makes difficult any generalization.

Remark \#1: in plane samples, a crack was beforehand made with a razor blade. Therefore, the crack initiation was not studied.

Remark \#2: in plane samples, the crack was made either in the thickness [21] or in the width [13], which leads to very different mechanical states at the crack tip.

Remark \#3: it should be noted that striations previously appeared in [28], but they were neither described nor commented.

\subsubsection{Loading conditions}

Most of the tests performed in the literature were conducted with a loading ratio, defined as the ratio of the minimum to the maximum loading, equal to zero. For relaxing tension, $\mathrm{R}_{F}=\frac{F_{\min }}{F_{\max }}=0$ and the corresponding displacements $\mathrm{U}_{\min }$ and $\mathrm{U}_{\max }$ increase during the test. For repeated tension, $\mathrm{R}_{U}=\frac{U_{\min }}{U_{\max }}=0$ and the corresponding forces $\mathrm{F}_{\text {min }}$ and $\mathrm{F}_{\max }$ decrease during the test. Le Cam et al. [16] investigated more complex loadings under prescribed displacement and/or angle: tension-compression $\left(\mathrm{R}_{U}<0\right)$, tension-tension $\left(0<\mathrm{R}_{U}<1\right)$, repeated torsion $\left(\mathrm{R}_{\theta}\right.$ $=0)$, fully-reversed torsion $\left(R_{\theta}=-1\right)$, repeated tension-torsion in opposite phases $\left(\mathrm{R}_{U}=0\right.$ and $\left.\mathrm{R}_{\theta}=0\right)$. Generally, frequencies are chosen to optimize the trade-off between fatigue test duration and self-heating level. In practice, this leads to a 
maximum frequency equal to a few Hertz. Thus, Flamm et al. [8] limit the selfheating to $45^{\circ} \mathrm{C}$ at the sample surface whereas Le Cam and Toussaint [13] and Le Cam et al. [16] limit the increase in the surface temperature at $20^{\circ} \mathrm{C}$ in order not to superimpose thermal damage to mechanical damage.

It is to note that the relevant temperature level to be considered is that of the crack tip zone, where the mechanism of crack growth takes place. In the Diabolo sample, at the crack tip of the macroscopic cracks, the temperature of the material should be lower than in the bulk. Unfortunately, as the crack tip shape and position evolve during the fatigue test, temperature measurement is not possible, even with non-contact measurement techniques.

Furthermore, deformation rates are quite different from one study to another. This is even the case for each study as the signal prescribed is sinusoidal. Muñoz-Mejia [22] did not investigate the effect of the loading level (tests were all performed at $\varepsilon_{\max }=90 \%$ ) but investigated the effects of temperature on the striation regime $\left(\right.$ at $60^{\circ} \mathrm{C}$ ). Finally, no information on loading conditions leading to striations are given in [8].

As a summary, experimental conditions strongly differ from one study to another, especially the sample geometry that induces different mechanical state at the crack tip. Consequently, whatever the results obtained, the generalization to fatigue of filled NR seems once again complicated.

\subsection{Results obtained}

The sample geometry was found to strongly influence the phenomenon of fatigue striation. For this reason, results are presented with respect to the sample geometry.

\subsubsection{Fatigue striation in plane samples}

Figure 1 describes the different geometries of plane sample used. Fig. 1(a) corresponds to a plane sample with a precut crack in the width direction. This type of geometry was used by Muñoz-Mejia [22] and Flamm et al. [8] with different dimensions: $20 \mathrm{~mm}$ in height, $220 \mathrm{~mm}$ in width and $1.4 \mathrm{~mm}$ in thickness for Muñoz-Mejia [22], a dumbbell geometry such as H2 sample in NFT46-002 standard for Flamm et al. [8]. In [13], the dimension was $20 \mathrm{~mm}$ in height, $5 \mathrm{~mm}$ in width and $2 \mathrm{~mm}$ in thickness. The precut crack was the thickness direction (see Fig. 1(a)). 
Failure surfaces obtained are schematized in Fig. 1(c), which corresponds to the A-A view in Fig. 1(a). What was observed by Munoz et al. [21] and Flamm et al. [8] appears inside the dotted lines black and red, respectively.

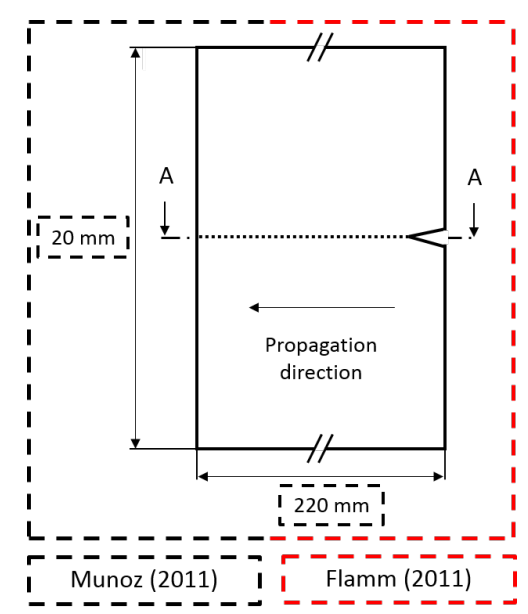

(a)

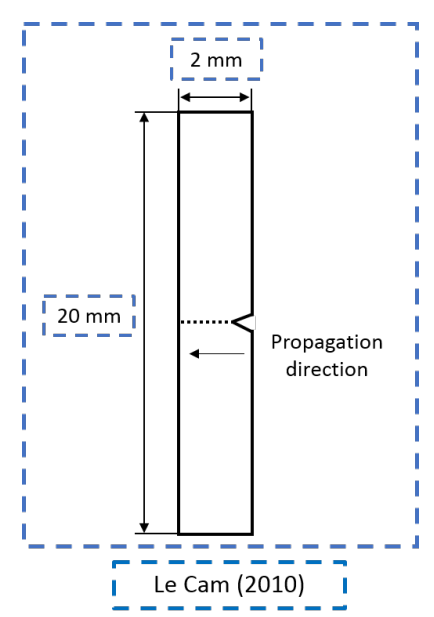

(b)

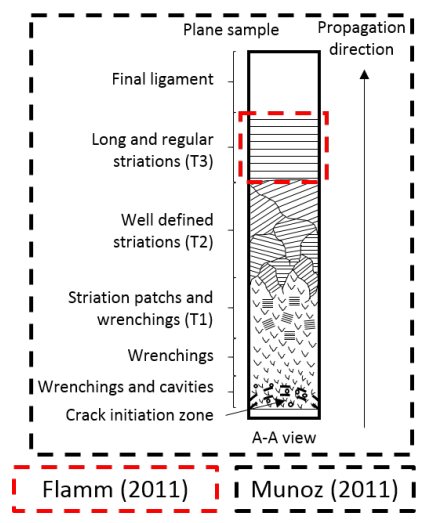

(c)

Fig. 1. Plane samples (a) pre-cut in the width (b) pre-cut in the thickness (c) A-A view of a plane sample after a fatigue test (from Muñoz-Mejia [22]).

The study by Muñoz-Mejia [22] is that revealing the largest striation shape diversity, taking place progressively during the fatigue experiment (see Fig. 3(c)).

First of all, the author describes a zone preceeding the striation zones during the crack propagation. This zone corresponds to crack initiation and propagation in every directions and exhibits numerous wrenchings. The crack tip in this zone follows a circular shape (see the circular dotted line). In this zone, many secondary cracks of about 10 microns in diameter and called cavitation by the author ini- 
tiate at the microscopic scale. It is worth noting that this is not observed once the crack propagates far from the initiation zone. Three striation typologies were distinguished by Muñoz-Mejia [22]. The first one corresponds to striation patches, which can be separated by wrenching zones. This striation typology is denoted T1 in the present paper. It corresponds to morphological group III in [22]. Their orientation differs from one patch to another. The second typology corresponds to well defined striations gathered in different zones orientated along slightly different directions. No wrenching was observed in this zone. This striation typology is denoted T2 in the present paper. It corresponds to morphological group IV in [22]. The third typology (T3) corresponds to long and regular striations occupying the whole sample width, i.e. from one side to the opposite one. This striation typology is denoted T3 in the present paper. It corresponds to morphological group $\mathrm{V}$ in [22]. The author classified striation topology according to the crack growth rate: typologies T1, T2 and T3 were observed for crack growth rate ranging between 1 and 2.3, 2.3 and 15, and 15 and $80 \mu \mathrm{m}$ per cycle, respectively. Increasing the test temperature at $60^{\circ} \mathrm{C}$ led to decrease the threshold in terms of crack growth rate at which each typology is observed. The authors did not link these results to SIC or crystallinity level, while increasing temperature decreases the crystallinity. Indeed, the change in the crack growth rate, in the conditions of striation occurrence, as well as the striation typology could be a consequence of the change in the crystallinity. Finally, Flamm et al. [8] performed test at $R_{\varepsilon}=0$ and only observed the third typology described by Muñoz-Mejia, corresponding to striation occupying the whole sample width. It can be explained by the difference in terms of loading conditions between the two studies.

Remark \#4: Le Cam and Toussaint [13] also used a plane sample, but precut in the width direction. According to the authors, this should be more representative as a cut in the thickness direction to investigate, during crack propagation, fatigue striation in the Diabolo sample. Nevertheless, it should be noted that a bias is induced by the proximity of the opposite surface, that could significantly affect the mechanical state at the crack tip. No quantitative information was provided by the authors.

\subsubsection{Fatigue striation in diabolo samples}

Fig.. 2(a) presents the Diabolo sample geometry. In Le Cam et al. [16], the sample dimension was $30 \mathrm{~mm}$ in height, $30 \mathrm{~mm}$ in diameter at the metallic insert and $20 \mathrm{~mm}$ in diameter at the middle. Flamm et al. [8] did not provide any dimension. Figure 2(b) gives a schematic view of a failure surface obtained with Diabolo samples. It is inspired from Figure 3 (page 3) in [13] and from Figure 13 (page 9) 


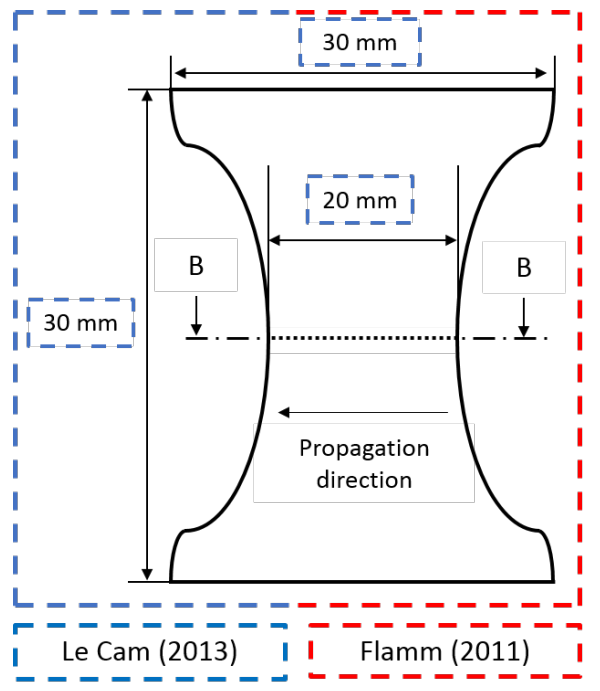

(a)

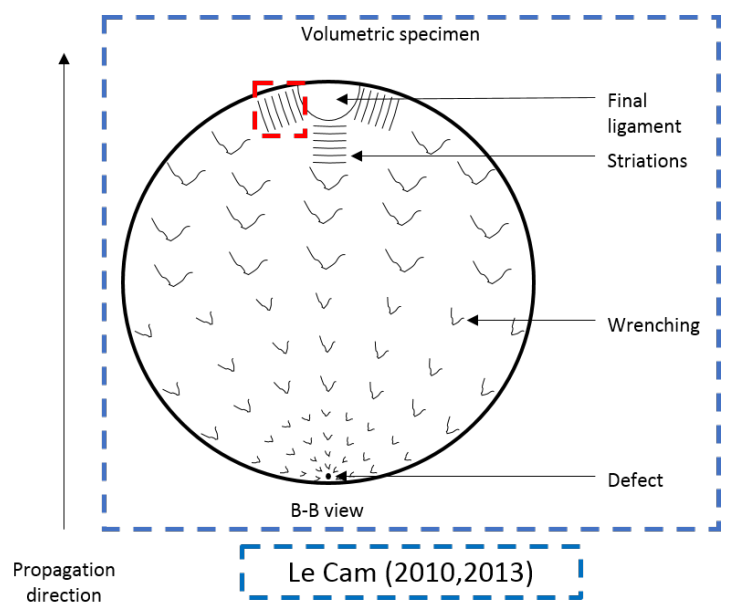

(b)

Fig. 2. Diabolo sample (a) Le Cam and Toussaint [13], Le Cam et al. [16], Flamm et al. [8] (b) B-B view of a Diabolo sample after a fatigue test from Le Cam and Toussaint [13], Le Cam et al. [16].

and Figure 15 (page 10) in [16].

According to the authors, crack initiates around a defect and propagates by generating small wrenchings following ellipsoidal zones around the defect [13]. These wrenchings exhibit the same morphology as those observed in plane samples. As the mechanism of wrenchings formation has already been established in [14], it is therefore not detailed here. According to the authors, striations are due to crack bifurcation due to the highly crystallized crack tip.

Note that striations were also observed for severe loadings under non relaxing conditions by Le Cam and Toussaint [13]. In this case, crack initiated below the metallic insert and striations formed as soon as the crack propagation started from several initiation zones, without forming any wrenching. It is worth noting that the mechanical state at the crack tip in a Diabolo sample is very different from that in a plane sample, close to the plane stress state.

Figure 3 summarizes the different striation morphologies reported in the literature. In the work by Muñoz-Mejia [22], the distance between two striations increases with the crack growth from 15 to $160 \mu \mathrm{m}$ : 15-20 $\mu \mathrm{m}$ in the T1 zone, 25-60 $\mu \mathrm{m}$ in the T2 zone and 50-160 $\mu \mathrm{m}$ in the T3 zone. Note that the height of the striations follow the same trend. The author highlighted the presence of crack bifurcations corresponding to a curvature of the decreasing slope of the striation. Le Cam 


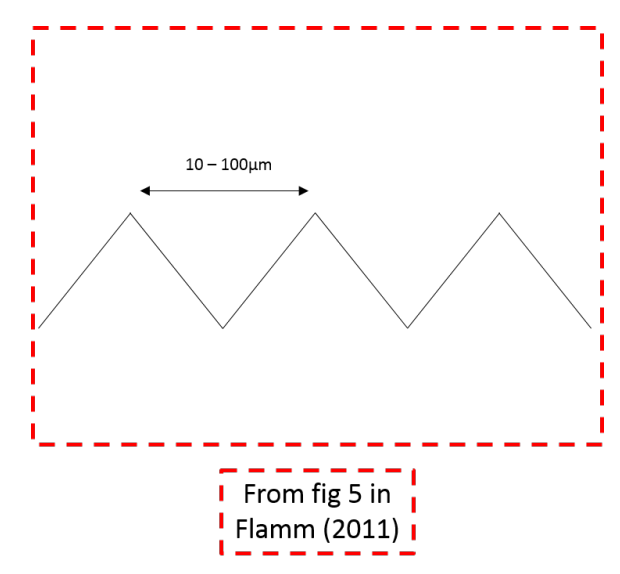

(a)

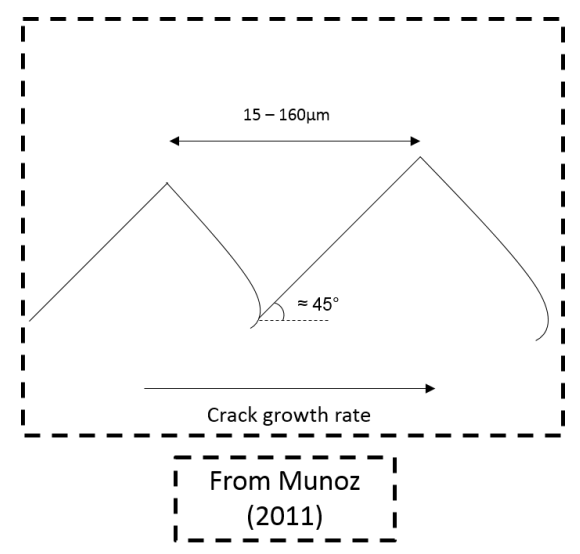

(b)

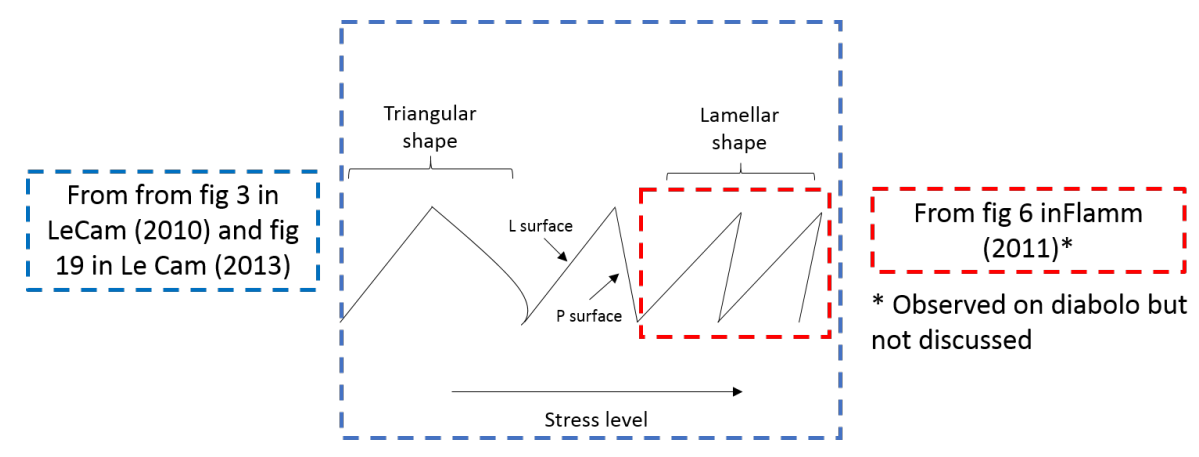

(c)

Fig. 3. Fatigue striation morphology

and co-workers described a striation as a triangular shape, composed of two perpendicular surfaces, denoted L and P. When the stress level increases, triangular morphology evolves into a lamellar morphology and the area of surface L increases, while that of the perpendicular surface $\mathrm{P}$ decreases until it becomes a line. Moreover, cracks are observed between the striations. It is to note that authors did not provide any metrological analysis. Flamm et al. [8] observed striations on both plane and Diabolo samples. The average distance between two striations in Diabolo sample can be deduced from Figure 6 (page 4) in [8]. It is approximatively $250 \mu \mathrm{m}$. For plane sample, it ranges between 10 and $100 \mu \mathrm{m}$. The authors did not further discuss striation morphology and its evolution in the crack propagation process. 


\subsection{Discussion and motivation for the present study}

As previously presented, only a few studies on the phenomenon of fatigue striation is available in the literature. Hence, several questions remain unanswered:

(i) Do fatigue striations bring new elements to better understand SIC effects in fatigue of NR?

On one hand, since fatigue striations were only observed on NR which crystallizes under tension, they are assumed to be due to SIC. On the other hand, lifetime reinforcement of $\mathrm{NR}$ is attributed to $\mathrm{SIC}[6,2,24]$. One can wonder what is the real relation between the mechanism of fatigue striation formation and the lifetime reinforcement.

(ii) What are the experimental conditions that lead to fatigue striation and which quantity(ies) drive(s) the formation of fatigue striations?

Results differ from one study to another so that no general conclusions on the occurrence of fatigue striation can be drawn. This is mainly explained by the differences between the material compositions, the sample geometries and the loading conditions applied in the studies. In [21], i.e. under plane stress, striations form for moderate loadings and the evolution of their typology is described with respect to the crack growth rate. The crack growth rate is not discussed as a consequence of SIC. According to the other authors, striations form only under severe loadings. Furthermore, experiments were generally carried out at $\mathrm{R} \geq 0$. For Le Cam and co-workers, striation occurs at a given stress level range: below the one leading to monotonic failure but above the one leading to wrenchings. No general answer on the quantity driving striation formation is provided in the literature and new fatigue tests are required to go further.

(iii) Crystallinity is a function of temperature and stretch [25]. At $60^{\circ} \mathrm{C}$, striations were still observed in plane sample [21]. At temperature higher than $80^{\circ} \mathrm{C}$ most of the crystallites are assumed to melt. Therefore, what would be the fracture surface obtained at temperatures superior to $60^{\circ} \mathrm{C}$, i.e. will striations still occur at very low crystallinity level?

To answer these questions, additional fatigue tests at different loading ratios and temperature are required. 


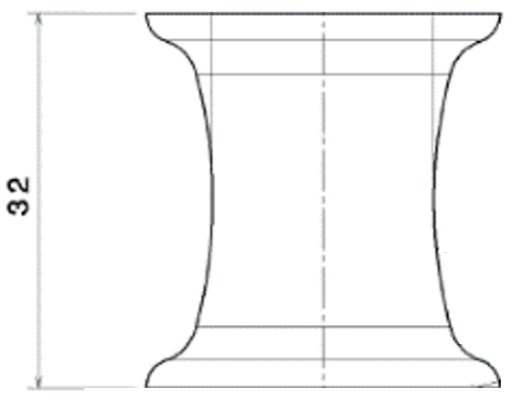

(a)

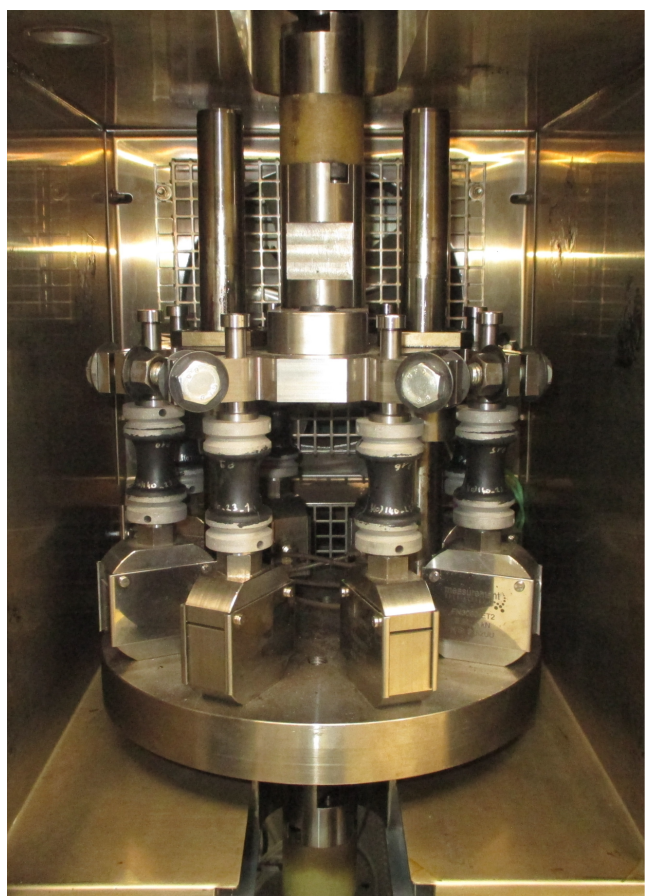

(b)

Fig. 4. Experimental setup : (a) diabolo sample; (b) home-made apparatus for testing eight samples simultaneously

\section{$3 \quad$ Experimental setup}

\subsection{Material and sample geometry}

The material considered in the present study is a natural rubber (cis-1,4 polyisoprene) vulcanised with sulphur and filled with carbon black aggregates. Table 2 summarizes its chemical composition.

In order to be representative of rubber parts, typically for anti-vibratory systems, a Diabolo sample is used. It is presented in Figure 4. As mentioned above, this geometry was firstly introduced by Beatty [3]. Its advantages have already been presented in Section 2.1.1. 
Table 2

\begin{tabular}{|c|c|}
\hline Ingredients & Quantity (phr) \\
\hline NR & 100 \\
Zinc oxide & 10 \\
Plasticizer & $<3$ \\
Carbon black & $20-30$ \\
Sulphur & 1.5 \\
Stearic acid & 2 \\
Antioxidant & $2-4$ \\
Accelerators & $2-4$ \\
\hline
\end{tabular}

Chemical composition of the natural rubber

\subsection{Loading conditions}

The fatigue tests were performed under prescribed displacement with a uni-axial MTS Landmark equipped with a home-made apparatus. The apparatus, presented in Fig. 4, enables us to test simultaneously and independently eight Diabolo samples, which strongly reduces the fatigue campaign duration. In order to investigate the influence of the loading on fatigue striation, different loading ratios $R_{\varepsilon}{ }^{4}$ were applied. They are presented with the corresponding minimum and maximum strains in Table 3 . We recall here that loading ratios inferior, equal and superior to zero correspond to tension-compression, repeated tension and tension-tension test, respectively. In practice, fatigue tests are carried out until failure at a given $\mathrm{R}_{\varepsilon}$ and the number of cycles at crack initiation is deduced from the evolution of the maximum force.

The frequency was chosen in such a way that the global strain rate $\dot{\varepsilon}$, i.e. whatever the signal shape, was kept constant and equal to a value between $1.8 \mathrm{~s}^{-1}$ and 2.4 $\mathrm{s}^{-1}$. Thus, the frequency varied from 1 to $4 \mathrm{~Hz}$, depending on the strain amplitude. This limited the material self-heating so that no thermal damage was added to the mechanical damage. In practice, this led to a maximal temperature reached at the sample surface equal to $40^{\circ} \mathrm{C}$. It should be noted that even though the temperature in the bulk material is much higher than in surface, convection occurs and limits the temperature raise, which is the case in the crack tip zone, where the mechanism of crack growth takes place.

$\overline{4 \mathrm{R}_{\varepsilon}}=\frac{\varepsilon_{\min }}{\varepsilon_{\max }}$ 


\begin{tabular}{|c|c|c|c|}
\hline $\mathrm{R}_{\varepsilon}$ & $\varepsilon_{\min }(\%)$ & $\varepsilon_{\max }(\%)$ & frequency $(\mathrm{Hz})$ \\
\hline \multirow{2}{*}{-0.25} & -18 & 72 & 2.5 \\
\hline & -25 & 100 & 1,5 \\
\hline \multirow{4}{*}{0} & 0 & 60 & 3 \\
\hline & 0 & 90 & 2.5 \\
\hline & 0 & 120 & 1.5 \\
\hline & 0 & 150 & 1.5 \\
\hline \multirow{4}{*}{0.125} & 8.75 & 70 & 4 \\
\hline & 12.5 & 100 & 2.5 \\
\hline & 17.5 & 140 & 2 \\
\hline & 21.5 & 172 & 1.5 \\
\hline \multirow{6}{*}{0.25} & 17.5 & 70 & 4 \\
\hline & 30 & 90 & 3 \\
\hline & 40 & 150 & 2 \\
\hline & 43 & 172 & 1.5 \\
\hline & 50 & 200 & 1.5 \\
\hline & 62.5 & 250 & 1 \\
\hline
\end{tabular}

Table 3

Uni-axial loading conditions

\subsection{Scanning Electron Microscopy}

Secondary electron images of fatigue striations are recorded with a JSM JEOL 7100 F scanning electron microscope (SEM). In addition, the SEM is coupled with an Oxford Instrument X Max Energy Dispersive Spectrometer of X-rays (EDS) and an Aztec software in order to determine the surface fracture composition, especially in the crack initiation zone. The material does not conduct sufficiently electrons. Therefore, the fracture surfaces to be analyzed were beforehand metallized by vapour deposition of a $2 \mathrm{~nm}$ thick $\mathrm{Au}-\mathrm{Pd}$ layer. 


\subsection{Optical Microscopy}

Optical microscopy is used to precisely measure the area of the fatigue striation surfaces. Keyence VHX 5000 optical microscope is used and equipped with two lens in the present to allow magnification from $\times 1$ to $\times 5000$. Unlike SEM, the fracture surfaces do not require any preparation. Altisurf 500 metrology station and its software PheNIX were used in order to collect striation 3D positions. Altimap V7 software was used to analyse data. The dimension of the zone of interest was $0,5 \times 2 \mathrm{~mm}^{2}$ and the surface of the measuring step was $5 \times 5 \mu \mathrm{m}^{2}$. No sample preparation was needed.

\section{Results}

In this section, fatigue striations are described with respect to their spatial distribution and typology. The effects of the macroscopic loading on fatigue striation occurrence and typology is then discussed.

\subsection{Spatial distribution of fatigue striations}

The spatial distribution of fatigue striations was investigated by using scanning electron and optical microscopy. An example is given in Figure 5 with the optical microscope. This figure depicts a typical fracture surface where striations are observed close to the final ligament. It should be noted that at the stage where they appeared, the stress level has increased and consequently its rate as well, as the tests are performed under prescribed displacement. Fatigue striations mainly appeared along the surface of the Diabolo sample and peopled the bulk when the loading applied at the microscopic scale increased. The lower the distance to the final ligament, the higher the striation number.

\subsection{Typology of fatigue striations}

Post-mortem analyses revealed that striations formed with respect to two regimes. This is highlighted in Figure 6(a).

The first regime (Regime 1) corresponds to small striation patches (see Fig. 6(a)) 


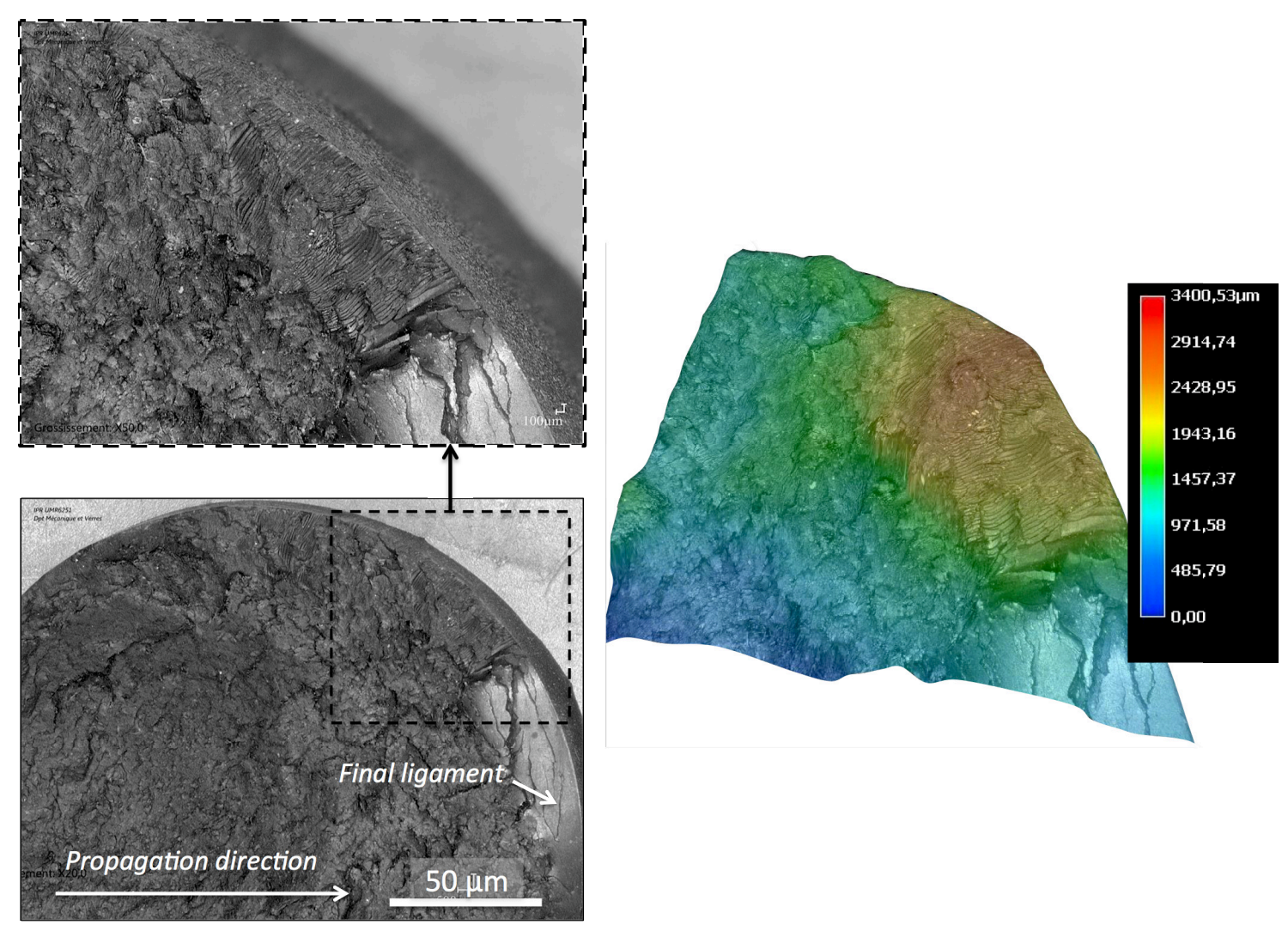

Fig. 5. Failure surface of a diabolo sample after a fatigue test at $\mathrm{R}_{\varepsilon}=0, \varepsilon_{\max }=150 \%$, $\mathrm{f}=1,5 \mathrm{~Hz}$. The color scale is for the relative elevation in $\mu \mathrm{m}$

with different orientations. Wrenchings can be observed in Regime 1, in between the patches. To our best knowledge, the present study is the first that reports striation formed in Regime 1 in a Diabolo sample. The relative distances between two striations in Regime 1 is small, in the order of magnitude of $20 \mu \mathrm{m}$. The stress level and crack growth rate are moderate and the Regime 1 corresponds to the transition between full wrenchings and full striations zones.

Regime 2 stands for well formed fatigue striations, where wrenchings are no longer observed (see Figure 6(b)). Regime 2 is the striation typology identified in the literature for Diabolo sample [13, 8, 16]. The zones where Regime 2 was observed were close to the final ligament, where the local stress can be considered as severe. The striations have a rather smooth surface. The relative distance between two striations in Regime 2 was higher than in Regime 1, in the order of magnitude of $100 \mu \mathrm{m}$. In Regime 2, the stress and the stress rate were higher than in Regime 1. Note that Regime 2 was always preceded by Regime 1. For the two regimes, fatigue striations are triangular. Contrary to Munoz et al. [21] who observed long (from on 


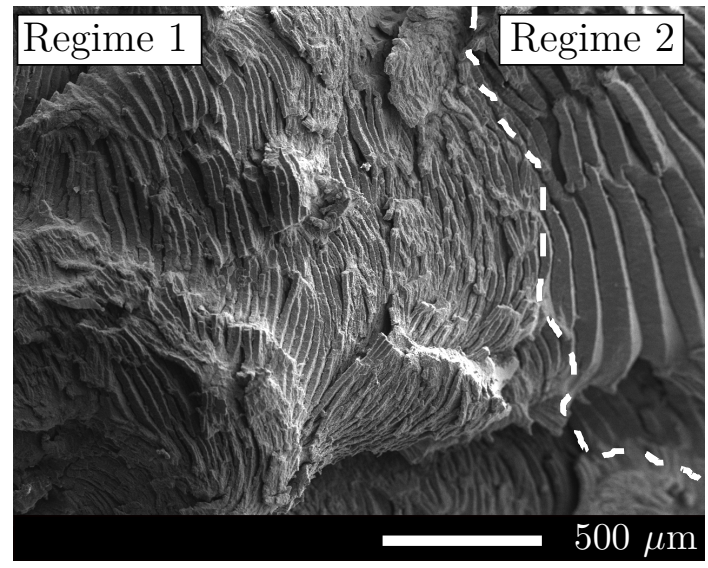

(a)

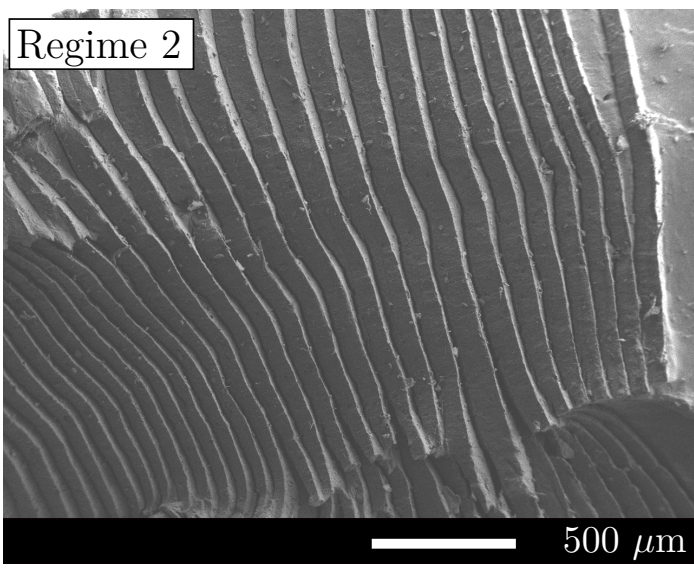

(b)

Fig. 6. SEM images of the two striation regimes (a) Regime 1 (b) Regime 2

side to the other one) and regular striations on plane samples, volumetric effects in the Diabolo sample do not enable them to form, due to the $3 \mathrm{D}$ effects at the crack tip and the fact that the crack does not propagate homogeneously along its curved tip. The third regime, corresponding to lamellar striations, has not been observed with the loading conditions applied in the present study.

\subsection{Linking fatigue striation to loading conditions}

In order to better understand the striation phenomenon and to discuss the role of SIC in the fatigue resistance of NR, striations have been linked to the loading conditions applied. Among the different diagrams commonly used to analyse fatigue results, the Haigh diagram provides the iso-lifetime curves in relation to the amplitude and the mean value of the loading, normally the stress. It is classically used to highlight the NR lifetime reinforcement obtained for non relaxing tension. In case of NR, a strong fatigue reinforcement is obtained for loadings applied at positive $\mathrm{R}$ ratios $[2,24]$. This result was firstly reported in [6] where the increase of the iso-lifetime curves begins at a loading ratio (in terms of strain) in the range of 0 to 0.125 , in other words for a positive value of $\varepsilon_{\min }$. In the present study, the Haigh diagram plots strains. This is the reason why the term pseudo Haigh diagram is used in the following. This representation have already been used to link fatigue damage at the macroscopic scale to loading conditions in [15] and [16], and to predict fatigue crack initiation from fatigue crack growth curves in [19].

Before classifying fracture surface zones, fatigue damages are presented at the 
macroscopic scale. They are reported in the pseudo Haigh diagram in Figure 7 with respect to the loading applied (see Table 3). The loading applied is defined in terms of the local strain, determined by using finite element calculations. Three types of fracture surface, regarding fatigue striation, have been identified:

- initiation in the surface vicinity and propagation of a crack in the median section. The crack propagates through the median section until sample failure,

- initiation in the surface vicinity and propagation of a crack bifurcating in the median section zone,

- initiation and growth of a crack below the metallic insert.

The three damage modes can lead to the same fatigue lifetime, depending on the loading ratio. Moreover, the two latter damage modes, obtained under non-relaxing loading conditions were not observed anymore at $90^{\circ} \mathrm{C}$. This demonstrates that fatigue damage modes are strongly governed by SIC. Finally, it should be noted that Le Cam [12] observed fatigue striations for the third damage mode (crack below the metallic insert).

A classification of fracture surface zones has been carried out in the same diagram:

(1) fracture surface with fatigue striation in Regime 1,

(2) fracture surface with fatigue striation in Regime 2. It should be noted that Regime 2 striation is consecutive to Regime 1. Therefore, fracture surfaces denoted (2) also exhibit Regime 1,

(3) fracture surface with no fatigue striation.

The case where no failure was observed after 1,5 millions cycles is denoted (4). The result obtained are first described according to the loading ratio $\mathrm{R}_{\varepsilon}$. The effect of the temperature is then discussed.

\subsection{1 $R_{\varepsilon}=0$ (repeated tension)}

Repeated tension corresponds to the most applied loading condition in fatigue of rubber. For this loading condition, fatigue striations were observed whatever the maximal strain applied. For low maximal strains, striations correspond to Regime 1. As the maximal strain increases, Regime 2 appears. Striation morphology remains triangular even close to the final ligament. No lamellar striation is observed. With the increasing maximum loading, both striations height and distance between two striations increases, as firstly quantified by Munoz et al. [21] for plane samples. 


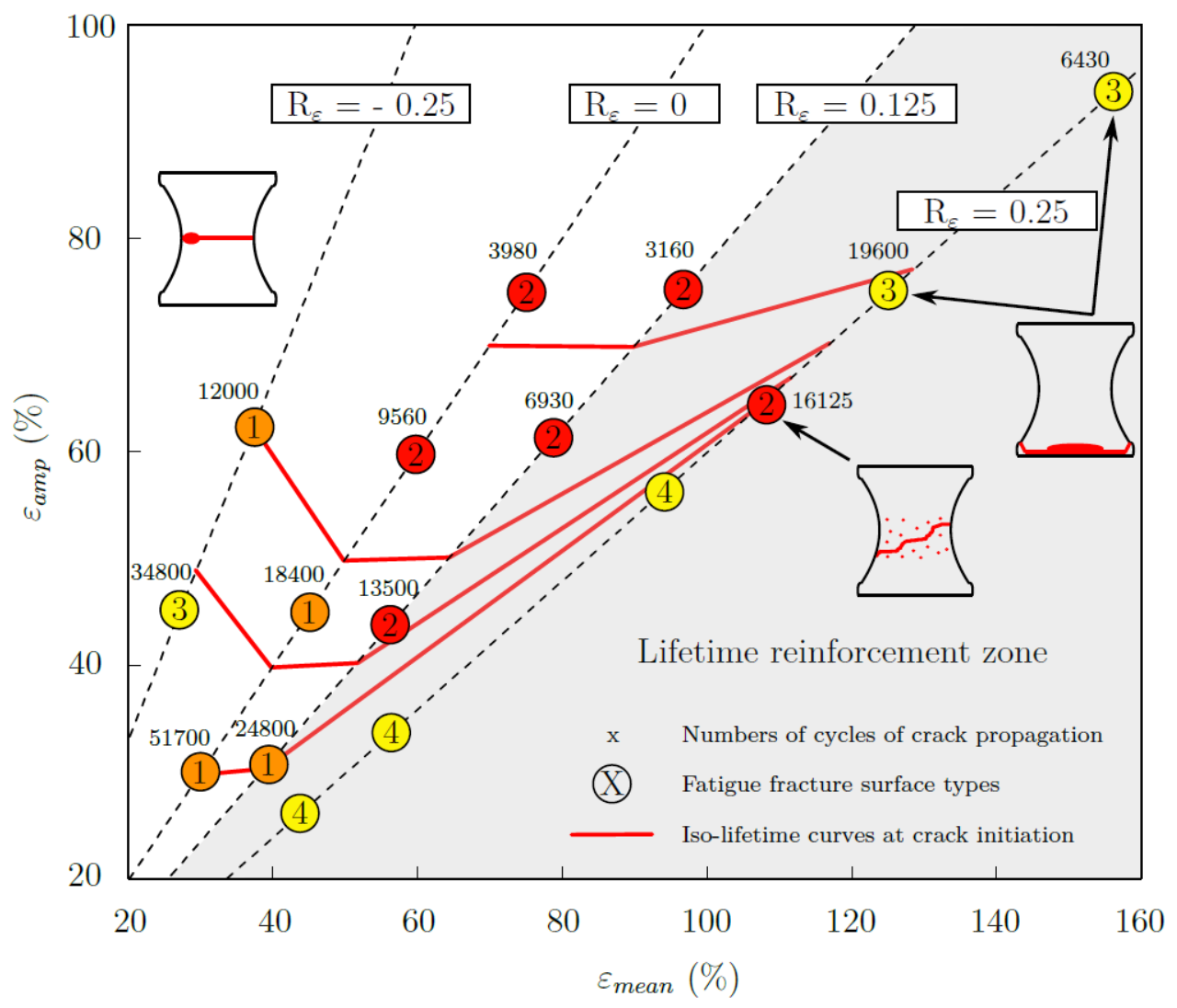

Fig. 7. Effect of loading conditions on the iso-fatigue lifetimes at crack initiation, the fracture surface typologies, the damage modes and the numbers of cycles of crack propagation. For a sake of confidentiality, the lifetime values are not provided.

\subsection{2 $R_{\varepsilon}>0$ (non relaxing tension)}

For non relaxing tension, the same comments can be drawn as for repeated tension. Nevertheless, Regime 2 appears at lower $\varepsilon_{\max }$. This can be deduced by considering iso- $\varepsilon_{\max }$ lines, perpendicular to the $\mathrm{R}_{\varepsilon}=0$ line. Therefore, $\varepsilon_{\min }$ influences the fatigue striation regime. According to Beurrot-Borgarino et al. [4], in such loading conditions, the crystallinity never goes back to zero during the fatigue test, which could influence the striation regime and promotes the occurrence of Regime 2 . It should be noted that for the highest loading level applied at $R_{\varepsilon}=0.25$, striations were not observed but the damage mode is different, i.e. the crack initiates and propagates below the metallic inserts. 


\subsection{3 $R_{\varepsilon}<0$ (tension-compression)}

Fatigue striation were also observed under tension-compression loading, but at higher $\varepsilon_{\max }$. In this case, the fact that crystallinity goes back to zero during the fatigue test should explain the occurrence of striation at higher $\varepsilon_{\max }$. It should be noted that Le Cam [12] ${ }^{5}$ observed the occurrence of a third regime, i.e. lamellar striations, for severe loading conditions that were not observed here.

\subsubsection{Effect of temperature}

At $23^{\circ} \mathrm{C}$, fatigue striation is found to form whatever the loading ratio applied. To investigate the effects of temperature on fatigue striation, and therefore the effect of SIC on their formation, fatigue tests described in Table 3 were reconducted at $90^{\circ} \mathrm{C}$. Literature suggests that no crystallites remain at such a temperature level $[27,26,1,18,23]$. Results obtained showed that neither striations nor wrenchings were observed, even at the highest maximal deformation applied. This confirms the role of SIC on wrenching and striation formation.

\section{Discussion}

The state of art presented in Section 2 highlighted that a number of questions on fatigue striation and the link with the role of SIC in fatigue remain unanswered. These questions are discussed in the following points:

- On one hand, fatigue striations have only been observed in crystallizable NR, this is why they were assumed to be due to SIC. On the other hand, lifetime reinforcement of $\mathrm{NR}$ is attributed to $\mathrm{SIC}[6,2,24]$. Hence, the question is to know if striations could be the signature of this lifetime reinforcement. The results obtained in the present study show that striations appear whatever the loading ratio applied (tension-tension, but also repeated tension and tension compression for which no fatigue life reinforcement is observed). Therefore, striations are the signature of SIC, but not of the lifetime reinforcement due to SIC. Note, however, that striations are more numerous under non-relaxing loading conditions.

- As striations did not appear when the tests were performed at $90^{\circ} \mathrm{C}$, this suggests that striations only form when a certain crystallinity level is reached.

$\overline{5}$ The $\mathrm{PhD}$ thesis from which was issued the paper by Le Cam et al. [16] 
- In [21], striations can form for moderate loadings in plane samples, i.e. under plane stress conditions, submitted to relaxing loadings. The striation typology and its evolution are discussed by the authors with respect to the crack growth rate. According to Flamm et al. [8] and Le Cam et al. [16], striations form under severe loadings in Diabolo samples, where the mechanical state at the crack tip is different from that in plane sample. Our investigations carried out with Diabolo samples with a wide range of loading ratios have shown that for negative loading ratios, striations (only Regime 1) appear at the highest $\varepsilon_{\max }$. For fully relaxing loadings $\left(\mathrm{R}_{\varepsilon}=0\right)$, the striation typology evolves from Regime 1 to Regime 2 when the maximum loading is increased. These results can be qualitatively put into perspective with crack propagation curves, see for instance [17], for which increasing the loading, i.e. the tearing energy, leads to an increase in the crack growth rate and to a striation typology evolution, especially the striation size. This unifies the conclusions drawn from results obtained for relaxing and fully relaxing loadings $\left(\mathrm{R}_{\varepsilon} \leq 0\right)$.

- For non relaxing loadings, Regime 2 occurs for a lower value of the maximum strain ${ }^{6}$ than under tension-compression or repeated tension. It should be noted that Regime 1 is only observed for the lowest maximum loading and lowest loading ratio (0.125). Hence, increasing the minimum value of the strain amplified the striation phenomenon and the occurrence of Regime 2. This is in a good agreement with the fact that under non-relaxing tension SIC effects are amplified and the lifetime reinforcement is observed. Again, analogy exists with the work by [17], who obtained crack growth curves at different loading ratios. ${ }^{7}$

He showed that increasing the loading ratio from zero decreases the crack growth rate at a given maximum tearing energy. Moreover, the threshold in terms of tearing energy below which no crack growth occurs increases with the increasing loading ratio. Also, the author stated that this crack growth resistance reinforcement is due to SIC. This is clearly the trend that we observed for non-relaxing loading conditions: below a certain maximum loading, no crack propagation occurred and the sample did not failed (see (4); only Regime 2 is observed. In this case, Regime 2 appears while the crack growth rate should be lower than that under relaxing loading. To go further on this point, the number of cycles corresponding to crack propagation $N_{p}$ have been reported in the pseudo Haigh diagram. It should be noted that the experiments were carried out at approximatively the same global mean

$\overline{6}$ In the Haigh diagram, the maximum strain corresponds to a line perpendicular to the $\mathrm{R}_{\varepsilon}=0$ line.

7 Lindley presented crack growth curves obtained for different loading ratios with unfilled NR, but he stated that filled NR should qualitatively behave in the same way. 
strain rate of $\dot{\varepsilon}= \pm 2 \mathrm{~s}^{-1}$. For relaxing and fully relaxing loadings, Regime 1 is obtained for the highest $N_{p}$, i.e. the lowest crack growth rate, and Regime 2 for the highest crack growth rate. For non-relaxing loadings, for instance at $\mathrm{R}_{\varepsilon}=0.125, N_{p}$ decreased when the loading level increased, which means that the crack growth rate increased, and the striation typology goes from Regime 1 to Regime 2. Regime 2 is obtained at $\mathrm{R}_{\varepsilon}=0.25$ at a higher $N_{p}$, i.e. at a lower crack growth rate, than at $R_{\varepsilon}=0.125$ at the same maximum loading. In order terms, at constant maximum loading, increasing the minimum loading decreases the crack growth rate. Our results suggest therefore that under non-relaxing loading the striation typology is rather governed by the loading ratio rather than the crack growth rate. These results highlight that crack propagation curves can be used to analyze qualitatively the signature of SIC on the failure surface with respect to the loading ratio. This is consistent with the work by [19] who predicted the iso-lifetime of natural rubber in the Haigh diagram from crack growth curves at different loading ratios.

\section{Conclusion}

Classically, SIC is assumed to be the main phenomenon involved in crack growth resistance of natural rubber. Under certain fatigue conditions, striations form on the failure surfaces of such a crystallizable rubber. Up to now, striations have not been observed in non-crystallizable rubbers. Investigating the link between SIC and striations could therefore provide additional information on SIC. The state of the art presented in the first part of the paper have shown that very few studies addressed such a topic and that they did not enable us to draw general conclusions on the role of SIC on the fatigue striation formation, which motivates new investigations. In the second part of the paper, uniaxial fatigue tests were carried out with Diabolo samples under a wide range of loading ratios and loading levels. Fracture surfaces were analyzed by using SEM. Results showed that two striation regimes occur. The first one corresponds to small striation patches with different orientations. Wrenchings can be observed in between them. Regime 2 stands for zones with large and well formed striations, where wrenchings are no longer observed. It is consecutive to Regime 1 and appears when the loading increases at the crack tip. Fatigue striations were observed for all the loading ratios applied, including relaxing loading conditions. They are therefore not the signature of SIC in the lifetime reinforcement. Nevertheless, increasing the minimum value of the strain amplifies the striation phenomenon and the occurrence of Regime 2. Results obtained have been qualitatively put into perspective with crack propagation curves provided in [17]. The analysis carried out unifies the results obtained in 
the literature for relaxing and fully relaxing loadings $\left(R_{\varepsilon} \leq 0\right)$ in the sense that increasing the loading, i.e. the tearing energy, leads to an increase in the crack growth rate and to a striation typology evolution, especially the striation size. For non-relaxing loadings, our results suggest that the striation typology may be governed by the loading ratio rather than the crack growth rate. Finally, as striations did not appear when the tests were performed at $90^{\circ} \mathrm{C}$, the formation of striation requires therefore that the material is crystallizing.

\section{Acknowledgements}

The authors thank the Cooper Standard France company for supporting this work and for fruitful discussions. The authors thank also the National Center for Scientific Research (MRCT-CNRS and MI-CNRS) and Rennes Metropole for financially supporting this work. SEM images were performed at CMEBA facility (ScanMAT, University of Rennes 1) which received a financial support from the European Union (CPER-FEDER 2007-2014).

\section{References}

[1] P. A. Albouy, J. Marchal, and J. Rault. Chain orientation in natural rubber, part i: The inverse yielding effect. The European Physical Journal E, 17(3): 247-259, Jul 2005. ISSN 1292-895X. doi: 10.1140/epje/i2004-10145-6. URL https://doi.org/10.1140/epje/i2004-10145-6.

[2] N. André. Critère local d'amorcage de fissures en fatigue dans un élastomère de type NR. Ph.d. thesis, Ecole Nationale Supérieure des Mines de Paris, 1999 .

[3] J. R. Beatty. Fatigue of rubber. Rubber Chemistry and Technology, 37:13411364, 1964.

[4] S Beurrot-Borgarino, Bertrand Huneau, Erwan Verron, and Pierre Rublon. Strain-induced crystallization of carbon black-filled natural rubber during fatigue measured by in situ synchrotron x-ray diffraction. International Journal of fatigue, 47:1-7, 2013.

[5] H. Bouasse and Z. Carrière. Courbes de traction du caoutchouc vulcanisé. Annales de la Faculté des Sciences de Toulouse, 5:257-283, 1903.

[6] S. M. Cadwell, R. A. Merril, C. M. Sloman, and F. L. Yost. Dynamic fatigue life of rubber. Industrial and Engineering Chemistry (reprinted in Rubber Chem. and Tech. 1940;13:304-315), 12:19-23, 1940. 
[7] J. H. Fielding. Flex life and crystallisation of synthetic rubber. Industrial and Engineering Chemistry, 35(12):1259-1261, 1943.

[8] M. Flamm, J. Spreckels, T. Steinweger, and U. Weltin. Effects of very high loads on fatigue life of $\mathrm{nr}$ elastomer materials. International Journal of Fatigue, 33(9):1189 - 1198, 2011. ISSN 0142-1123. doi: http://dx.doi.org/10.1016/j.ijfatigue.2011.03.008. URL //www.sciencedirect.com/science/article/pii/S014211231100082X.

[9] John Gough. A description of a property of caoutchouc, or indian rubber. Memories of the Literacy and Philosophical Society of Manchester, 1:288-295, 1805.

[10] J. P. Joule. On some thermodynamic properties of solids. Phil Mag 4th, 14: $227,1857$.

[11] J. R. Katz. Naturw, 4:169, 1925.

[12] J.-B. Le Cam. Endommagement en fatigue des elastomères. Ph.d. thesis, Université de Nantes, École Centrale de Nantes, décembre 2005.

[13] J.-B. Le Cam and E. Toussaint. The mechanism of fatigue crack growth in rubbers under severe loading: the effect of stress-induced crystallization. Macromolecules, 43:4708-4714, 2010.

[14] J.-B. Le Cam, B. Huneau, E. Verron, and L. Gornet. Mechanism of fatigue crack growth in carbon black filled natural rubber. Macromolecules, 37:50115017, 2004.

[15] J.-B. Le Cam, E. Verron, and B. Huneau. Description of fatigue damage in carbon black filled natural rubber. Fatigue and Fracture of Engineering Materials \& Structures, 31:1031-1038, 2008.

[16] Jean-Benoît Le Cam, Bertrand Huneau, and Erwan Verron. Fatigue damage in carbon black filled natural rubber under uni- and multiaxial loading conditions. International Journal of Fatigue, 52(Supplement C):82 - 94, 2013. ISSN 0142-1123. doi: https://doi.org/10.1016/j.ijfatigue.2013.02.022. URL http://www.sciencedirect.com/science/article/pii/S0142112313000716.

[17] PB Lindley. Relation between hysteresis and the dynamic crack growth resistance of natural rubber. International Journal of Fracture, 9(4):449-462, 1973.

[18] J. Marchal. Cristallisation des caoutchoucs chargés et non chargés sous contrainte : Effet sur les chaînes amorphes. Ph.d. thesis, Université Paris XI Orsay, France, 2006.

[19] W. V. Mars. Computed dependence of rubber's fatigue behavior on strain crystallization. Rubber Chemistry and Technology, 82(1):51-61, 2009. doi: 10.5254/1.3557006. URL https://doi.org/10.5254/1.3557006.

[20] W. V. Mars and A. Fatemi. Factors that affect the fatigue life of rubber: A literature survey. Rubber Chemistry and Technology, 77(3):391-412, 2004. doi: 10.5254/1.3547831. URL https://doi .org/10.5254/1.3547831. 
[21] L. Munoz, L. Vanel, O. Sanseau, P. Sotta, D. Long, L. Odoni, and L. Guy. Fatigue crack growth dynamics in filled natural rubber. Plastics, Rubber and Composites, 41(7):273-276, 2012. doi: 10.1179/1743289812Y.0000000013. URL http://dx.doi.org/10.1179/1743289812Y.0000000013.

[22] Luisa Muñoz-Mejia. Étude expérimentale des mécanismes d?endommagement par fatigue dans les élastomères renforcés. Ph.d. thesis, Université Claude Bernard Lyon 1, 2011.

[23] J Rault, J Marchal, P Judeinstein, and PA Albouy. Stress-induced crystallization and reinforcement in filled natural rubbers: $2 \mathrm{~h} \mathrm{nmr} \mathrm{study.} \mathrm{Macro-}$ molecules, 39(24):8356-8368, 2006.

[24] N. Saintier. Prévisions de la durée de vie en fatigue du NR, sous chargement multiaxial. Ph.d. thesis, École Nationale Supérieure des Mines de Paris, 2000.

[25] S. Trabelsi, P.-A. Albouy, and J. Rault. Stress-induced crystallization around a crack tip in natural rubber. Macromolecules, 35:10054-10061, 2002.

[26] S. Trabelsi, P.A. Albouy, and J. Rault. Crystallization and melting processes in vulcanized stretched natural rubber. Macromolecules, 36(20):7624-7639, 2003.

[27] L. R. G. Treloar. The Physics of Rubber Elasticity. Oxford University Press, Oxford, 1975.

[28] B. Wang, H. Lu, and G. Kim. A damage model for the fatigue life of elastomeric materials. Mechanics of Materials, 34:475-483, 2002. 


\section{List of Figures}

1 Plane samples (a) pre-cut in the width (b) pre-cut in the thickness (c) A-A view of a plane sample after a fatigue test (from Muñoz-Mejia [22]).

2 Diabolo sample (a) Le Cam and Toussaint [13], Le Cam et al. [16], Flamm et al. [8] (b) B-B view of a Diabolo sample after a fatigue test from Le Cam and Toussaint [13], Le Cam et al. [16].

$3 \quad$ Fatigue striation morphology

4 Experimental setup : (a) diabolo sample; (b) home-made apparatus for testing eight samples simultaneously

5 Failure surface of a diabolo sample after a fatigue test at $\mathrm{R}_{\varepsilon}=$ $0, \varepsilon_{\max }=150 \%, \mathrm{f}=1,5 \mathrm{~Hz}$. The color scale is for the relative elevation in $\mu \mathrm{m}$

6 SEM images of the two striation regimes (a) Regime 1 (b) Regime 2

$7 \quad$ Effect of loading conditions on the iso-fatigue lifetimes at crack initiation, the fracture surface typologies, the damage modes and the numbers of cycles of crack propagation. For a sake of confidentiality, the lifetime values are not provided. 